\title{
Investigación en la acción. Un ejemplo de estudio experimental en el mercadeo de servicios
}

\begin{abstract}
RESUMEN
En el artículo se muestra un ejemplo de aplicación de la metodología de la investigación en la acción. Se presenta el diseño y resultados de un estudio referido a la telefonía móvil celular en el aspecto de decisiones tarifarias, se trata de comparación de medias para muestras dependientes, midiendo el efecto en el tráfico telefónico celular entrante debido al cambio de estrategia tarifaria, por parte del operador de telefonía celular. En el caso bajo estudio, el comportamiento de un cliente con reducido tráfico de llamadas entrantes hacia teléfonos celulares manifiesta un problema inicial que afecta las ganancias de la empresa. Dentro de diversas alternativas, se plantea que el cambio de modalidad tarifaria para el cliente celular incrementaría la recepción de llamadas, elevando el tráfico telefónico entrante y los ingresos. Para corroborar esta hipótesis se definió una muestra aleatoria de 25 clientes a los que se les comunicó y se les aplicó un nuevo modelo tarifario, en forma restringida durante un mes, haciendo un seguimiento de los resultados a través del sistema informático. Este tipo de experimento se denomina diferencia de medias de poblaciones relacionadas, donde se considera la misma muestra en diferentes momentos.
\end{abstract}

Palabras clave: investigación, diseño experimental, estrategia tarifaria

RESEARCH IN ACTION. AN EXAMPLE OF EXPERIMENTAL STUDY IN MARKETING SERVICES

\section{ABSTRACT}

The article shows an example of application of the methodology of action research. We present the design and results of a study based on the mobile phone in the aspect of pricing decisions, it's comparison of means for dependent samples, measuring the effect on incoming cellular telephone traffic due to changing pricing strategy, by cellular operator. In case, the customer behavior with traffic reduced incoming calls to cell phones manifests an initial problem that affected the company's earnings. Among various alternatives, the mode change for the customer tariff suggests increase receiving phone calls, raising the incoming telephone traffic and revenue. To corroborate this hypothesis we defined a random sample of 25 clients who applied a new tariff model, on a restricted basis for a month, tracking the results through the computer system. This type of experiment is called the mean difference related populations, which is considered the same sample at different moments.

Keywords: research, experimental design, pricing strategy

\section{INTRODUCCIÓN}

Cuando se inicia el crecimiento de los servicios de comunicaciones (telefonía, Internet, cable), los nuevos operadores de telecomunicaciones invierten en incremento de capacidad y la implantación de mejoras tecnológicas, cuyo éxito depende de la madurez de los mercados locales [3]. La dirección de estas empresas encuentra un entorno deprimido y un modelo de crecimiento agotado. El incipiente desarrollo de los mercados nacionales, caracterizados por la poca capacidad de pago de la ínfima cantidad de clientes, inexistencia de masa crítica mínima para la operación rentable de la empresa, elevada población económicamente activa sin actividad laboral sostenida, fuerte economía informal maneja la reducida oferta de los servicios, conformaban un mercado distorsionado donde es difícil identificar segmentos, estudiar al cliente y prever conductas ante la nueva oferta de servicios.

El incipiente desarrollo de estos mercados, que impiden prever el comportamiento y respuesta de los clientes ante los nuevos servicios de comunicaciones y ante las novedosas estrategias que se diferenciaban de lo que, tradicionalmente, el cliente estaba acostumbrado a recibir. Dado que las técnicas de los estudios de mercado no ofrecían información importante sobre servicios desconocidos, se consideró que el uso de otras técnicas como el diseño de experimentos sobre una muestra estadística generaría datos relevantes acerca de la respuesta del universo poblacional, o sea, el mercado de los clientes de telecomunicaciones, la adecuada información facilitará la toma de las mejores decisiones en el nuevo marketing de servicios.

\section{CASO ESTUDIO DE COMPORTAMIENTO DE CLIENTE}

\subsection{Antecedentes del uso del servicio}

La empresa de comunicaciones donde se realiza el estudio, mantiene una elevada inversión en telefonía celular, pero el número de clientes y el tráfico no crece. La dirección plantea el

* Doctor en Ingeniería Industrial, Magíster en Administración, Ingeniero Industrial, Economista Profesor en la Facultad de Ingeniería Industrial-UNMSM. E-mail: aacevedo@speedy.com.pe, aacevedo@industrial.unmsm.pe

** Magíster en Dirección de Empresas, Ingeniero Industrial, estudios de Derecho. Profesora en UPG Facultad de Ingeniería de Sistemas e Informática UNMSM. E-mail: klinares@speedy.com.pe

*** Doctor en Ingeniería Industrial, Profesor en la Facultad de Ingeniería Industrial, Departamento de Producción y Gestión Industrial de la UNMSM. E-mail: orestescachay@yahoo.es 
objetivo comercial de elevar las ventas mediante el incremento del número de clientes y un mayor uso del servicio.

El aumento de ventas se relaciona a la política publicitaria mediante tácticas promocionales de corto plazo para que el cliente adquiera el equipo terminal de comunicación donde la conexión telefónica es (aparentemente) gratis. El costo de la línea se cubre con un cargo fijo mensual.

El mayor uso del servicio es un problema de mayor complejidad [2], ya que depende del comportamiento del usuario ante el servicio, de su percepción como elemento de comunicación costoso que se debe usar ante urgencias o accidentes, o como medio de comunicación barato común para todas las circunstancias diarias. Para alcanzar un cambio de perspectiva [1] ante el servicio y una modificación de conducta se plantearon diversas propuestas de mercadeo que implicaban elevado costo por la contratación de "creativos" de las agencias de publicidad que consideraban que el efecto emulación del "cholo ascendente", el concepto de moda en aquellos tiempos, y la contratación de personajes televisivos, futbolistas y vedettes era la clave para incentivar un mayor uso por los clientes.

\subsection{La tarifa como determinante de la conducta del cliente}

Por el lado de la empresa, se consideró que la variable precio era determinante del uso del servicio, por lo que la alternativa elegida fue diseñar un nuevo sistema de tarifas que reemplazaría al antiguo y tradicional sistema tarifario. El nuevo sistema denominado "calling party pay" (cpp) o "el que llama paga" implicaría que el cliente celular pagaría por toda llamada saliente y las llamadas entrantes contestadas serían sin costo para el celular, la llamada recibida es pagada por el que está originando la llamada (sea otro celular o un teléfono fijo).

El sistema de tarifas tradicional en celular es el denominado "mobile party pay" (mpp) o "celular paga todo" donde el cliente celular paga toda llamada saliente y entrante. El usuario que origina la llamada no paga nada (cero costo si es teléfono fijo) o duplica el pago (costo de llamada saliente si es celular). Este sistema originaba que el cliente celular mantenga el teléfono apagado y solo lo prendía para efectuar llamadas, ya que recibir llamadas (equivocadas, no deseadas, intrascendentes) elevaban el costo mensual del servicio.

\subsection{Comportamiento del cliente celular}

Se realizó un estudio de las cuentas celulares y se determinó que las siguientes variables sintetizaban el modo de emplear el servicio (datos modificados para no mostrar información reservada).

- El promedio de minutos utilizados por cliente celular es cien minutos por mes, aproximadamente.

- El cliente prende su equipo para efectuar llamadas y el resto del tiempo lo mantiene apagado. En ese caso, se calcula que el $10 \%$ de los minutos de tráfico son por llamadas entrantes.

- La variación de la facturación por consumo mensual es mínima, porque el cliente maneja su límite de crédito (si el cliente utiliza sus minutos libres, entonces deja de llamar).

- Cuando un celular llama a otro celular, el pago es doble (ambos clientes pagan por la llamada). Cuando un teléfono fijo llama a un celular no paga nada.

\section{MÉTODO DE INVESTIGACIÓN EXPERIMENTAL}

Para el estudio del caso se emplea el esquema conceptual de la investigación en la acción, planteado por Lewin en el estudio de modelos sociotécnicos y desarrollado en aspectos de sistemas empresariales con la metodología de los sistemas blandos [4].

\subsection{Diseño experimental}

El modelo general del diseño experimental se muestra en la figura 1, aunque el diseño específico a ser elegido ha de estar condicionado por el enfoque seleccionado, el problema a investigar, el contexto que rodea la investigación, el alcance del estudio, las hipótesis formuladas. Desde la perspectiva del investigador, las variables son de dos tipos, la variable independiente susceptible de ser manipulada por el investigador, la variable dependiente que mide el efecto de manipular la variable independiente [5].

\section{Las variables del estudio}

La variable independiente se caracteriza por:

- No depende del fenómeno bajo estudio.

- Se manipula, por grados: presencia-ausencia, intensidad o por modalidad: verbal y escrita.

- Se operacionaliza convirtiendo el concepto abstracto en hecho real. El concepto se traslada 


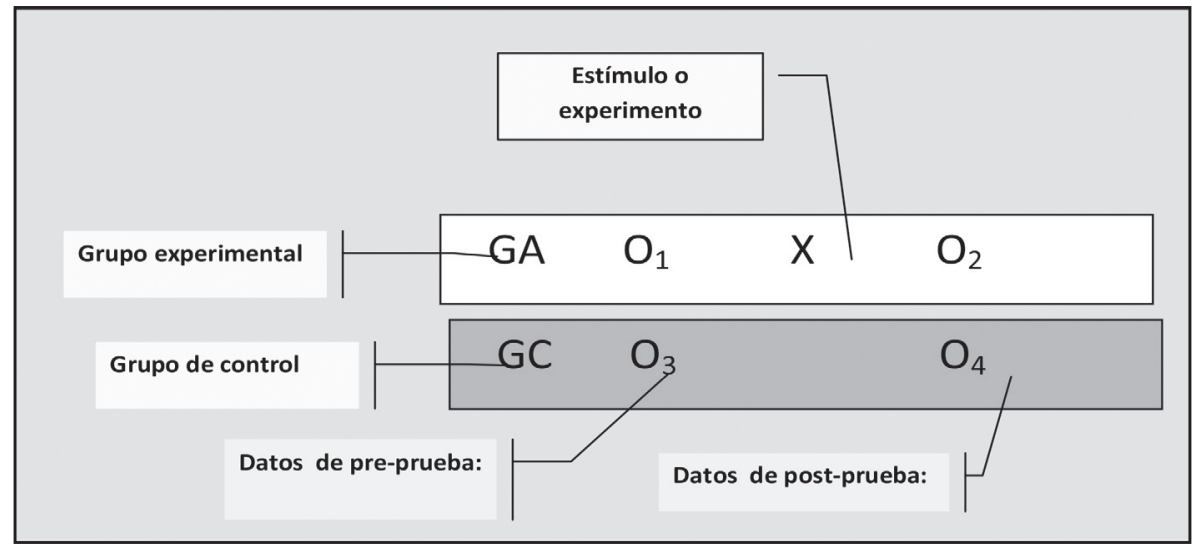

Figura 1. Modelo general del diseño experimental.

Fuente: Elaboración propia en base a Fernández et al. (2006).

a una serie de operaciones y actividades concretas.

La variable dependiente se caracteriza por:

- Depende de la variable independiente, es efecto, no causa.

- Se mide considerando dos grupos mínimo (de experimento y de control).

- Puede contener más de dos grupos aunque siempre habrá un grupo de control.

\section{Validez del estudio}

La validación puede ser de dos tipos:

- Interna, respecto a la elección de grupos y el control.

- Externa, para generalizar resultados y aplicarlo a otros grupos.

La invalidez surge por dos causas:

- Interna: por el experimento mal ejecutado o por experimentador en sí.

- Externa: por los efectos reactivos que genera, la interacción entre pruebas, incapacidad de replicación.

\subsection{Diseño de la investigación en estrategia tarifaria}

Diseño de la prueba previo al lanzamiento comercial. La nueva estrategia tarifaria cpp generó elevado rechazo de los gerentes ya que dejaban de recibir el pago por llamadas recibidas, y no se convencían que habría un cambio de conducta en el uso del servicio, ya que el cliente mantendría el celular prendido recibiendo llamadas que, de otra manera, serían llamadas perdidas. Además se argumentaba que el cliente fijo no llamaría al celular por lo costoso del minuto de tráfico.

Dentro del diseño tarifario se esperaba, con esta medida, los clientes reciban llamadas, elevando así el tráfico entrante. Por otro lado, existía el riesgo que la medida no genere cambio alguno, ya que los teléfonos fijos (que antes no pagaban por llamar a celular) ahora deberían pagar un promedio de setenta centavos de dólar, lo cual no facilitaría que las llamadas a celulares aumenten.

Dado que no existían antecedentes históricos, ni en el país, ni en el extranjero sobre cambios de hábitos de consumo en servicios de comunicación, se propuso un diseño experimental sobre el cual se plantearían las hipótesis y se verificarían los efectos sobre una muestra reducida y controlada de usuarios del servicio.

El diseño experimental comprende los siguientes aspectos:

- GA: Grupo aleatorio. El grupo de prueba se conformó sobre una muestra aleatoria de 25 clientes, a los que se les brindó información sobre el cambio de modalidad tarifaria.

- X: diseño de prueba. Los participantes GA recibieron la información siguiente:

- Las llamadas entrantes a su celular no serán pagadas por ellos.

- El costo de la llamada será cubierta por el que llama.

- Si la llamada viene de su casa, el costo será cubierto por su línea familiar fija. 
- Si la llamada viene de otro celular, dicho celular paga.

- Si el cliente llama a otro celular, solo uno de los dos, paga.

- El cliente fijo que llama sabe que pagará el costo de la llamada.

- 01: Datos de pre-prueba. Los datos pre prueba corresponde a la facturación normal de un período mensual completo, que refleja el tráfico y el comportamiento en el uso del servicio, de los clientes del grupo GA.

- 02: Datos de post-prueba. Los datos posteriores a la prueba corresponde a la facturación de un mes completo, que refleja los cambios en el tráfico y en el uso del servicio, de los clientes del grupo GA.

A pesar que se espera la generación de profusa información relacionada a comportamiento en llamadas entrantes y salientes, solamente se consideraron las llamadas entrantes.

\subsection{Formulación del experimento}

\section{Situación Problemática}

La fuente principal de ingresos de la empresa es el uso del servicio o tráfico telefónico. Este tráfico se mide en minutos utilizados por mes.

El tráfico total por cada cliente se define por la fórmula siguiente:

\author{
$\mathrm{TT}=\mathrm{TS}+\mathrm{TE}$ \\ Donde: \\ TT: tráfico total \\ TS: Tráfico saliente \\ TE: Tráfico entrante
}

\section{EI Problema de Investigación.}

Responde a la siguiente pregunta de investigación:

- ¿De qué manera el modelo tarifario cpp afecta la facturación del servicio celular?

Como problemas secundarios se definen:

- ¿Cómo el modelo cpp incidirá en aumentar el uso del servicio por parte del cliente celular?

- ¿El cliente cambiará su conducta ante llamadas entrantes?

\section{La hipótesis del Problema de Investigación}

Según el diseño experimental se considera una hipótesis nula y una hipótesis alternativa.

Según la hipótesis nula, el tráfico entrante preprueba y post-prueba son iguales. El cambio tarifario no afecta el tráfico entrante telefónico celular entrante.

Según la hipótesis alternativa, el tráfico post-prueba es mayor al tráfico pre-prueba.

\section{Justificación del diseño experimental}

- Se intenta mostrar el efecto del nuevo modelo tarifario cpp en una muestra aleatoria de clientes, antes de decidir su lanzamiento masivo.

- Se busca conocer si el nuevo modelo tarifario cpp incide en el uso y tráfico del servicio celular, de manera que justifique el costo de su implantación y desarrollo comercial.

- Los resultados de la prueba han de facilitar la decisión de cambio de modelo de tarifas. La intención es mostrar elementos de juicio que permitan la toma de decisión más adecuada, respecto a la implantación, o no, del nuevo modelo tarifario.

\section{LA INVESTIGACIÓN EXPERIMENTAL Y RE- SULTADOS}

\subsection{Aplicación de la prueba}

\section{Diseño y tipo de Investigación}

Se plantea un experimento para comparar el comportamiento de tráfico entrante en dos períodos mensuales. Se comparan las medias de minutos de tráfico entrante con muestra dependiente en dos diferentes momentos del tiempo (misma muestra $\mathrm{GA}$ en dos diferentes momentos).

\section{Conceptos estadísticos}

Unidad de análisis: cliente de telefonía celular.

Variable: La cantidad de minutos de tráfico entrante durante un mes.

Dato: cantidad de minutos de tráfico entrante de un cliente durante un mes del período.

Instrumento de medición: Central telefónica (Switch) que registra CDR (call data record) acumulados del mes, en el sistema informático de facturación de tráfico.

Población: Total clientes de telefonía celular activos en un mes, período 1995-1996. 
Muestra: Grupo de clientes de telefonía celular, registrados en el sistema informático comercial en el período contable previo. Tamaño de muestra: 25 clientes. Selección de muestra: Aleatoria (al azar).

\section{Técnica de recolección de Datos}

A un grupo de clientes, elegidos al azar, se les envía una carta explicándole el cambio del sistema de tarificación de sus llamadas celulares, detallando las ventajas y las implicancias generales en su facturación. Se le solicita su autorización para incluirlo en la muestra.

De todos los que respondieron, se eligieron solamente 25 , se les envió instrucciones adicionales sobre las diferencias de mpp y cpp, aunque sin especificarles ni sugerir la manera en que deben administrar el on/off (prendido/apagado) de su equipo y si deben responder o no a las llamadas.

De los registros históricos del sistema informático comercial-facturación, se recuperaron los datos de tráfico y de facturación de los clientes de la muestra, se tomó un mes típico, elegido al azar entre los más recientes.

Posterior a la pruebas, se toman los datos del último mes, se recuperó el tráfico efectuado con los criterios del nuevo modelo cpp. Se registró el tráfico total y el tráfico entrante de los veinticinco clientes.

\subsection{Resultados de la prueba}

Según los resultados, se ha de verificar la hipótesis de investigación, brindando elementos de juicio para la implantación del nuevo modelo de tarificación CPP. La comunicación a los clientes, tomaría como base las instrucciones enviadas a los clientes de la muestra.

Datos de tráfico recolectados. Los datos de tráfico entrante se miden en minutos por mes. Se recolectó la información para el grupo aleatorio seleccionado.

En la tabla 1 se muestra:

CLIE-CEL: Ios 25 clientes celulares de la muestra.

TRAF-MPP: los minutos de tráfico entrante, por cliente, por el mes XX de 1995. Este tráfico corresponde a la tarifa MPP, donde el celular paga toda llamada saliente y entrante.

TRAF-CPP: los minutos de tráfico entrante, por cliente, por el mes YY de 1996. Este tráfico corresponde a la tarifa CPP en prueba, donde el celular paga solo las llamadas salientes.
Tabla 1. Tráfico entrante de muestra

\begin{tabular}{|c|c|c|}
\hline $\begin{array}{l}\text { CLIE-CEL } \\
\text { Cliente celular }\end{array}$ & $\begin{array}{c}\text { TRAF-MPP } \\
\text { Min entrantes } \\
\text { mes XX }\end{array}$ & $\begin{array}{c}\text { TRAF-CPP } \\
\text { Min entrantes } \\
\text { mes YY }\end{array}$ \\
\hline 1 & 11 & 12 \\
\hline 2 & 10 & 20 \\
\hline 3 & 11 & 14 \\
\hline 4 & 15 & 30 \\
\hline 5 & 18 & 33 \\
\hline 6 & 9 & 20 \\
\hline 7 & 18 & 22 \\
\hline 8 & 5 & 16 \\
\hline 9 & 14 & 33 \\
\hline 10 & 20 & 29 \\
\hline 11 & 14 & 14 \\
\hline 12 & 9 & 14 \\
\hline 13 & 11 & 12 \\
\hline 14 & 21 & 31 \\
\hline 15 & 9 & 21 \\
\hline 16 & 14 & 14 \\
\hline 17 & 12 & 35 \\
\hline 18 & 11 & 27 \\
\hline 19 & 20 & 40 \\
\hline 20 & 13 & 15 \\
\hline 21 & 18 & 25 \\
\hline 22 & 10 & 11 \\
\hline 23 & 12 & 20 \\
\hline 24 & 18 & 32 \\
\hline 25 & 17 & 22 \\
\hline
\end{tabular}

Fuente: Elaboración propia

\subsection{Estudio estadístico}

Prueba de Hipótesis

\section{$1^{\circ)}$ Hipótesis}

$$
\begin{array}{ll}
\mathrm{H} 0: & \mu 1=\mu 2 \\
\mathrm{H} 1: & \mu 1<\mu 2
\end{array}
$$

Donde:

$\mu 1$ : Media del tráfico entrante de la población, bajo la modalidad tarifaria MPP.

$\mu 2$ : Media del tráfico entrante de la población, bajo la modalidad tarifaria CPP.

Es equivalente a:

$\mathrm{HO}: \mu \mathrm{D}=0$

$\mathrm{H} 1: \mu \mathrm{D}<0$ 
Donde:

$\mu \mathrm{D}=$ media de las diferencias.

\section{$2^{\circ}$ ) Nivel de significación y Nivel de confianza} (n.s. y n.c.)

Nivel de significación $\quad \alpha=0.05$

Nivel de confianza $1-\alpha=0.95$

$3^{\circ}$ ) Función pivot:

$$
t=\frac{\bar{D}-\mu_{D}}{s_{D} / \sqrt{n}}
$$

Donde:

$\overline{\mathrm{D}}=$ promedio de las diferencias $=-222 / 25=-8.88$

$\mathrm{S}_{\mathrm{D}}=$ desviación estándar de diferencias $=$

$$
\sqrt{\frac{2}{\mathbf{S}_{\mathbf{D}}}}=\sqrt{(27066 / 600)=6.72}
$$

$\mathrm{n}=$ tamaño de la muestra $=25$

$$
\mathrm{t}=\frac{-8.88-0}{6.72 / \sqrt{25}}=-6.61
$$

\begin{tabular}{|c|c|c|c|c|c|c|c|c|c|}
\hline & & \multicolumn{5}{|c|}{ Diferencias relacionadas } & \multirow{3}{*}{$\begin{array}{c}\mathrm{t} \\
\text { Media } \\
\text { Superior }\end{array}$} & \multirow{3}{*}{$\begin{array}{c}\text { gl } \\
\begin{array}{c}\text { Desviación } \\
\text { típ. }\end{array} \\
\text { Inferior }\end{array}$} & \multirow{3}{*}{$\begin{array}{c}\text { Sig. } \\
\text { (bilateral) } \\
\text { Error típ. } \\
\text { de la media } \\
\text { Superior }\end{array}$} \\
\hline & & \multirow{2}{*}{$\begin{array}{l}\text { Media } \\
\text { Inferior }\end{array}$} & \multirow{2}{*}{$\begin{array}{c}\text { Desviación } \\
\text { típ. }\end{array}$} & \multirow{2}{*}{$\begin{array}{l}\text { Error típ. } \\
\text { de la media }\end{array}$} & \multicolumn{2}{|c|}{$\begin{array}{l}95 \% \text { Intervalo de } \\
\text { confianza para la } \\
\text { diferencia }\end{array}$} & & & \\
\hline & & & & & Superior & Inferior & & & \\
\hline Par 1 & $\begin{array}{l}\text { MPP min } \\
\text { entrantes - } \\
\text { CPP min } \\
\text { entrantes }\end{array}$ & -8.880 & 6.716 & 1.343 & -11.652 & -6.108 & -6.611 & 24 & .000 \\
\hline
\end{tabular}

$\left.4^{\circ}\right)$ Cálculo t mediante SPSS: Ver tabla 2.

Tabla 2. Prueba de muestras relacionadas

$\left.5^{\circ}\right)$ Prueba t para muestras relacionadas:

Figura 2. Aceptación o rechazo de hipótesis-

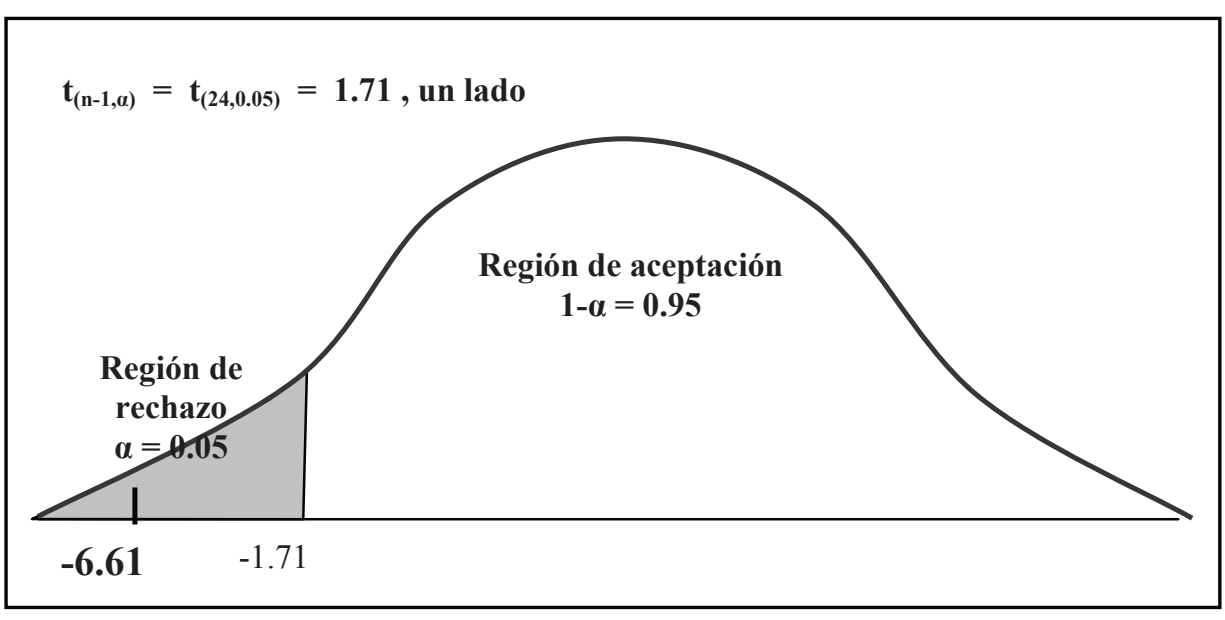

$\left.6^{\circ}\right)$ Decisión

$\alpha=0.05, \mathrm{gl}=24$

Valor $t_{t}=-1.71$ (un lado)

$t_{c}=-6.61$
Se rechaza la hipótesis nula a nivel de significación 0.05 .

Se acepta Hipótesis alternativa. 


\subsection{Resultados de la investigación experimental}

Según los resultados de la prueba de hipótesis, a un nivel de significación de 0.05 , no es posible aceptar la hipótesis de que el tráfico de minutos entrantes tiene igual media, sin interesar el modo de tarificación de dicho tráfico.

Alternativamente, no se puede rechazar la hipótesis que el tráfico valorizado con el modelo de tarificación cpp calling party pay, posea una media superior al tráfico valorizado con el modelo de tarificación mpp mobile party pay.

- Se rechaza la Hipótesis nula a un nivel de significación de 0.05 .

- Se acepta Hipótesis alternativa un nivel de significación de 0.05 .

\section{CONCLUSIONES}

En el estudio se presenta un caso de diseño de experimento estadístico aplicado a un problema empresarial del campo del mercadeo en telecomunicaciones, con las siguientes conclusiones.

1. El caso es desarrollado en un medio económico deprimido y de baja capacidad de consumo, muestra la utilidad del diseño de investigación para estudiar conducta del cliente con fines de toma de decisiones tarifarias.

2. El caso se refiere al cambio de modalidad tarifaria efectuada por la empresa de Teléfonos. El problema inicial era el reducido tráfico de llamadas entrantes a los teléfonos celulares, lo que reducía la rentabilidad de la empresa. Dentro de diversas alternativas, se determinó que el cambio de modalidad tarifaria para el cliente celular incrementaría su recepción de llamadas, lo que elevaría el tráfico telefónico entrante y los ingresos. Se definió una muestra aleatoria de 25 clientes a los que se les comunicó y se les aplicó el nuevo modelo tarifario, en forma restringida, realizando seguimiento de los resultados.

3. El sector de telecomunicaciones e información, caracterizado por su dinámica de cambio, con elevadas tasas de crecimiento, con innovación elevada y convergente, integración de sectores de negocio y tecnologías, genera nuevas situaciones problemáticas y nuevos retos, los que habrán de abordarse con enfoques novedosos e integración de conocimiento desde diferentes ramas del saber.

4. El nuevo modelo de tarificación representó un cambio sustantivo en el tráfico de telefonía celular entrante. Se implementó nuevo modelo de tarifas cpp.

5. Se modificó el comportamiento de uso de servicio celular de los clientes.

\section{REFERENCIAS BIBLIOGRÁFICAS}

[1] Acevedo Borrego, Adolfo (2010). Informe Técnico de proyecto "“'Las dimensiones del modelo conceptual 4D para la resolución de problemas", investigación exploratoria y descriptiva FII UNMSM. Centro de Investigación FII-UNMSM, Perú.

[2] Ackoff, Russell (2000). Recreación de las corporaciones. Un diseño organizacional para el siglo XXI. Ed. Oxford University Press. México.

[3] Checkland \& Scholes (1994). La Metodología de los Sistemas Suaves de Acción. Noriega Editores. México.

[4] Duncan, W. Jack (2000). Las ideas y la práctica de la administración, Los principales desafíos en la era moderna, Oxford University Press, México.

[5] Hernández Sampieri, Fernández, Baptista (2010). Metodología de la investigación. Quinta edición. Ed. Mcgraw-Hill, Chile. 